\title{
RISK ASSESSMENT OF A RFID - GSM BASED LOCK SYSTEM USING FMECA TECHNIQUE
}

\author{
S. O. Anaza \\ R\&D Department \\ Power Equipment and Electrical \\ Machinery Development Institute \\ Okene, Kogi State, Nigeria
}

\author{
J. D. Jiya \\ EEE Department \\ Abubakar Tafawa Balewa \\ University Bauchi-Nigeria
}

\author{
Y. S. Haruna \\ EEE Department \\ Abubakar Tafawa Balewa \\ University Bauchi-Nigeria
}

\begin{abstract}
Security systems are necessary everywhere especially in Banks, houses, offices etc. An RFID, GSM and Keypad Lock System is a multiprotocol device designed, constructed and customized to combine the security feature in RFID, Global System for Mobile communication (GSM) and password for the three components of access control which are identification, authentication, and authorization. The device was a series system (a failure of a unit result into failure of the system), therefore reliability analysis was conducted to reduce the failure rate using Failure Mode and Effects and criticality Analysis (FMECA). The aim of this paper is to present the reliability analysis of this security system. Each component/unit will be analyzed, depending on the degree; numbers were allotted for severity, occurrence and detection, and subsequently, the risk priority number (RPN) will be obtained for each unit and subsequently for the system before and after an action to increase its reliability. With the reliability measures, The result obtain shows that the severance of the system reduces by $\sim 16.4$, occurrence by $50 \%$ and detection by $\sim 11 \%$. The RPN also reduces by $\sim 68 \%$.
\end{abstract}

Keyword: FMECA, RPN, RFID, GSM, severity, occurrence and detection

\section{INRODUCTION}

Failure Mode and Effects Analysis (FMEA) is a proactive process aimed to evaluate a system, design, process and service for possible ways in which failures can occur [1]. An FMECA is generated from an FMEA by adding a criticality figure of merit. It is a technique used to identify, prioritize, and eliminate potential failures from the system, design or process before they reach the customer [2], it is used to resolve potential problem in a system during the design stage. FMECA can be required and applied during all stages of projects: at early design phase, during the detailed design and being the device in operation [3]. These analyses are performed for reliability, safety, and supportability information. The FMECA version is more commonly used and is more suited for hazard control. Terms and rules of safety analysis for electronic component are well described in well known ISO/IEC international standard series and technical reports [4]. A RFID-GSM base lock system is a security device designed, constructed and customized for a lock system. It has four security features which are: RFID system, the GSM module, password (keypad) and alarming mechanism (buzzer). The latter was additional security feature for notification. Each of this security features are used for three basic component of access control (identification, authentication and confirmation). Other component of the lock system are microcontroller and liquid crystal display (LCD), shown in figure 1.

The Microcontroller Controls the operation of the system while the GSM Module Send 4 digit code generated by the microcontroller to the person after the tag has been read successfully. The Buzzer notify any closer person any attempted intruder, LCD display any recommended information and the Key Pad will be use to enter the code after text message containing code is send to GSM of authorized user. The RFID reader reads the ID number from passive Tag and sends it to the microcontroller for confirmation,

From the literature reviewed, [5] highlighted the analysis of failure events observed in DC brush motors using the FMECA Technique. [6] performed a Failure Modes Effect and Criticality Analysis (FMECA) on a PV system through the use of an octopus diagram for functional analysis of the system. [7] identified, analyzed and evaluated the potential risks of unexpected failures occurring in 
rolling stock using a failure mode, effects and criticality analysis-based approach. [8] represented a generic process of FMECA for centrifugal pump failures and a case study on centrifugal pump failure cost estimation actual and after implementation of optimum strategies of maintenance. [9] Conducted research on reliability of transformer and proposed Failure Modes and Effects Analysis (FMEA) technique to increase the reliability and economic value. [10] conducted a high-level failure modes and effects analysis to characterize potential hazards from compressed-hydrogen fuel cell vehicles and identify potential safety issues for Federal Motor Vehicle Safety Standards, the National Highway Traffic Safety Administration in Washington DC. [11] in their research identified and eliminate current and potential problems from a manufacturing process of cylinder head in the company through the application of Failure Mode and Effects Analysis (FMEA).

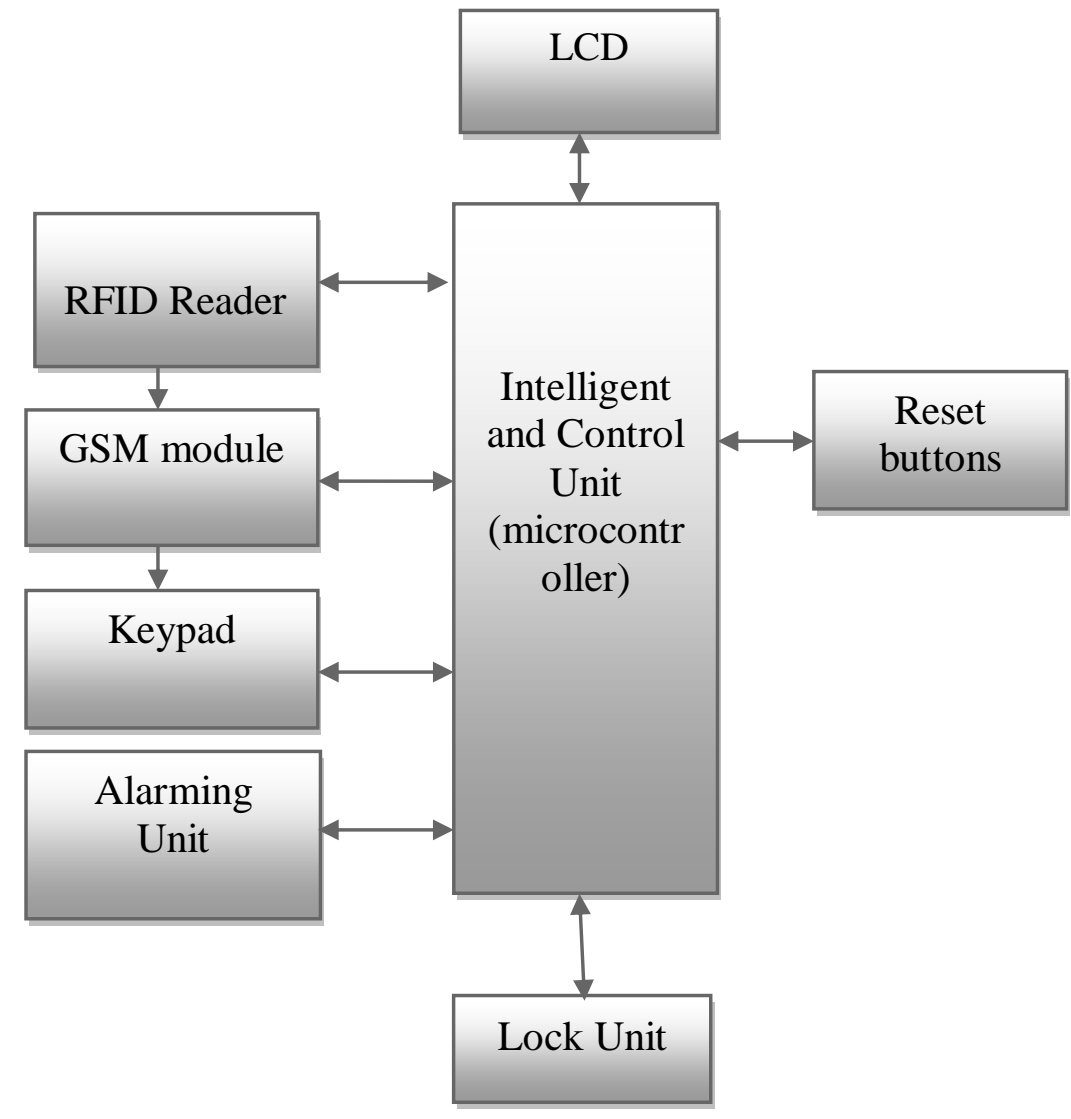

Figure 1: Block Diagram of the proposed Lock svstem

Everything that can fail, shall fail [12]. Due the important of security system, the reliability of such security lock system is as important as the security system itself. In most cases, developers of lock system ignore the risk assessment. These necessitate the needs to conduct reliability analysis on any designed lock system.

This paper presents the risk assessment of an RFID - GSM Based Lock System Using FMECA Technique by diligently studying the potential effect of fault, occurrence and possible detection. Depending on the degree, numbers were allotted and subsequently, the risk priority number (RPN) was obtained for each unit. Been a series system (a failure of a unit result into failure of the system), necessary actions were taken to reduce the RPN

\section{FUNDAMENTAL CONCEPT OF FMECA}

The following are the definition of some basic terms related to FMECA according to [13]. (i) Failure: Termination of the ability of an item to perform a required function. (ii) Failure mode: Manner in which an item fails. (iii) Failure cause and/or mechanism: Cause or sequence of causes that initiate a process (mechanism) that leads to a failure mode over a certain time. The most likely causes of 
the failure mode are listed under "Possible failure causes". (iv) Failure effects: Consequence of a failure mode in terms of the operation, function or status of the item.

The following are definition of terms related to FMECA according to [14]. (i) Severity: Severity is an assessment of the seriousness of the effect of the potential failure mode to the next component, subsystem, system or customer if it occurs. Severity applies to the effect only. A reduction in Severity Ranking index can be effected only through a design change. Severity Ranking is shown in Table 1. (ii) Occurrence (Event frequency): Occurrence is how frequently a specific failure cause/ mechanism are projected to occur. The likelihood of occurrence ranking number has a meaning rather than a value. Occurrence Ranking is shown in Table 2. (iii)
Detection: Detection is the ability to detect the cause/mechanism/weakness of actual or potential failure. In Design FMECA, this must occur before the component, subsystem, or system is released for production. In Process/Service FMECA it must occur in time to prevent distribution in case of a product or catastrophe in case of an Asset / Maintainable Unit. In order to achieve a lower ranking, generally the planned control (e.g. preventative activities) has to be improved. Detection Ranking is shown in Table 3. (iv) Risk priority Number (RPN): The Risk Priority Number is the product of the Severity (S), Occurrence (O), and Detection (D) ranking. It is a measure of design risk.

$R P N=S \times O \times D$.

Table 1: Table of Severity

\begin{tabular}{|c|c|c|c|}
\hline Codes & Classificati & & Example \\
\hline 10 & $\begin{array}{l}\text { Hazardous } \\
\text { Warning }\end{array}$ & Without & Very High Ranking - Affecting safe operation. \\
\hline 9 & $\begin{array}{l}\text { Hazardous } \\
\text { Warning }\end{array}$ & With & Regulatory non compliance \\
\hline 8 & Very High & & $\begin{array}{l}\text { Product becomes inoperable, with loss of function - Customer Very Much } \\
\text { Dissatisfied }\end{array}$ \\
\hline 7 & High & & Product remain operable but loss of performance - Customer Dissatisfied \\
\hline 6 & Moderate & & Product remain operable but loss of comfort/convenience - Customer Discomfort \\
\hline 5 & Low & & $\begin{array}{l}\text { Product remain operable but loss of comfort/convenience - Customer Slightly } \\
\text { Dissatisfied }\end{array}$ \\
\hline 4 & Very Low & & Nonconformance by certain items - Noticed by most customers \\
\hline 3 & Minor & & Nonconformance by certain items - Noticed by average customers \\
\hline 2 & Very Minor & & Nonconformance by certain items - Noticed by selective customers \\
\hline 1 & None & & No Effect \\
\hline
\end{tabular}

Source: [11]

Table 2: Table of occurrence

\begin{tabular}{lll}
\hline Code & Classification & Example \\
\hline 10 and 9 & Very High & Inevitable Failure \\
8 and 7 & High & Repeated Failures \\
6 and 5 & Moderate & Occasional Failures \\
4,3 and 2 & Low & Few Failures \\
1 & Remote & Failure Unlikely \\
\hline
\end{tabular}

Source: [11]

Table 3: Table of Detection

\begin{tabular}{|c|c|c|}
\hline Detection & Rank & Criteria \\
\hline Extremely likely & 1 & $\begin{array}{l}\text { Can be corrected prior to prototype/ Controls will almost certainly } \\
\text { detect }\end{array}$ \\
\hline Very High Likelihood & 2 & $\begin{array}{l}\text { Can be corrected prior to design release/Very High probability of } \\
\text { detection }\end{array}$ \\
\hline High Likelihood & 3 & Likely to be corrected/High probability of detection \\
\hline Moderately High Likelihood & 4 & Design controls are moderately effective \\
\hline Medium Likelihood & 5 & Design controls have an even chance of working \\
\hline
\end{tabular}




\section{International Journal of Engineering Applied Sciences and Technology, 2021 \\ Vol. 5, Issue 10, ISSN No. 2455-2143, Pages 44-54 \\ Published Online February 2021 in IJEAST (http://www.ijeast.com)}

$\begin{array}{lll}\text { Moderately Low Likelihood } & 6 & \text { Design controls may miss the problem } \\ \text { Low Likelihood } & 7 & \text { Design controls are likely to miss the problem } \\ \text { Very Low Likelihood } & 8 & \text { Design controls have a poor chance of detection } \\ \text { Very Low Likelihood } & 9 & \text { Unproven, unreliable design/poor chance for detection } \\ \text { Extremely Unlikely } & 10 & \text { No design technique available/Controls will not detect }\end{array}$

Source: [11]

\section{RISK ASSESSMENT OF RFID - GSM BASED LOCK SYSTEM.}

The following assumptions were made in order to complete this analysis.

(i) In situations where there are several failure results for one component failure mode, the most severe failure mode was documented in the analysis.

(ii) Single component failures only were investigated where possible, otherwise, failure of a unit was considered [15]. (iii) [11] and [16] were the documents used as the basis for the definition of the FMECA and component failure modes.

Depending on the degree, numbers were allotted for severance, occurrence and detection, and subsequently the risk priority number was obtained for each unit. Been a series system (a failure of a unit result into failure of the system), necessary action was taken to reduce the RPN. The analyses were presented in tables 4 to 10 . The DFMECA of the system is presented in table 11

Table 4: FMECA of power supply unit

\begin{tabular}{|c|c|c|c|c|c|c|c|c|c|c|c|c|c|}
\hline \multirow{2}{*}{$\begin{array}{l}\text { Parts of } \\
\text { power supply } \\
\text { unit }\end{array}$} & \multirow{2}{*}{$\begin{array}{l}\text { Failure } \\
\text { Mode }\end{array}$} & \multirow{2}{*}{$\begin{array}{l}\text { Effects(s } \\
\text { ) of } \\
\text { Failure }\end{array}$} & \multirow[b]{2}{*}{$\mathbf{S}$} & \multicolumn{4}{|c|}{ Risk rating } & \multirow[b]{2}{*}{$\begin{array}{l}\mathbf{R P} \\
\mathbf{N}\end{array}$} & \multirow[b]{2}{*}{$\begin{array}{l}\text { Action } \\
\text { s } \\
\text { Taken }\end{array}$} & \multicolumn{4}{|c|}{ Revised risk } \\
\hline & & & & $\begin{array}{l}\text { Cause(s) } \\
\text { of Failure }\end{array}$ & $\mathbf{O}$ & $\begin{array}{l}\text { Fault } \\
\text { Detectio } \\
\text { n }\end{array}$ & D & & & $\bar{S}$ & $\mathbf{O}$ & D & $\begin{array}{l}\mathbf{R} \\
\mathbf{P} \\
\mathbf{N}\end{array}$ \\
\hline $\begin{array}{l}\text { electrical } \\
\text { Power supply } \\
\text { from mains }\end{array}$ & $\begin{array}{l}\text { Failure } \\
\text { of power } \\
\text { from } \\
\text { mains }\end{array}$ & $\begin{array}{l}\text { loss of } \\
\text { power } \\
\text { supply to } \\
\text { the entire } \\
\text { system }\end{array}$ & 8 & $\begin{array}{l}\text { Load } \\
\text { shedding } \\
\text { fault, } \\
\text { system } \\
\text { maintenan } \\
\text { ce }\end{array}$ & 8 & $\begin{array}{l}\text { Extremel } \\
\text { y } \\
\text { Unlikely }\end{array}$ & 10 & 640 & $\begin{array}{l}\text { Battery } \\
\text { backup } \\
, . \\
\text { Indicat } \\
\text { or for }\end{array}$ & 2 & 2 & 2 & 8 \\
\hline Transformer & $\begin{array}{l}\text { open } \\
\text { circuit, } \\
\text { short } \\
\text { circuit }\end{array}$ & $\begin{array}{l}\text { loss of } \\
\text { power } \\
\text { supply to } \\
\text { the entire } \\
\text { system }\end{array}$ & 8 & $\begin{array}{l}\text { Manufact } \\
\text { urer } \\
\text { defect, } \\
\text { over } \\
\text { loading, } \\
\text { ageing }\end{array}$ & 2 & $\begin{array}{l}\text { Design } \\
\text { controls } \\
\text { have an } \\
\text { even } \\
\text { chances }\end{array}$ & 5 & 80 & $\begin{array}{l}\text { main } \\
\text { power } \\
\text { supply }\end{array}$ & 2 & 2 & 2 & 8 \\
\hline Rectifier & $\begin{array}{l}\text { open } \\
\text { circuit, } \\
\text { short } \\
\text { circuit }\end{array}$ & $\begin{array}{l}\text { loss of } \\
\text { power } \\
\text { supply to } \\
\text { the entire } \\
\text { system }\end{array}$ & 8 & $\begin{array}{l}\text { Manufact } \\
\text { urer } \\
\text { defect, } \\
\text { over } \\
\text { loading, } \\
\text { ageing }\end{array}$ & 3 & $\begin{array}{l}\text { Design } \\
\text { controls } \\
\text { have an } \\
\text { even } \\
\text { chances }\end{array}$ & 5 & 120 & & 2 & 2 & 2 & 8 \\
\hline $\begin{array}{l}\text { Voltage } \\
\text { regulator and } \\
\text { other } \\
\text { component }\end{array}$ & $\begin{array}{l}\text { open } \\
\text { circuit, } \\
\text { short } \\
\text { circuit, } \\
\text { Output } \\
\text { struck, } \\
\text { input } \\
\text { struck, } \\
\text { Average }\end{array}$ & $\begin{array}{l}\text { Unfiltere } \\
\mathrm{d} \text { and } \\
\text { unregulat } \\
\text { ed power } \\
\text { supply }\end{array}$ & 7.8 & $\begin{array}{l}\text { Manufact } \\
\text { urer } \\
\text { defect, } \\
\text { over } \\
\text { loading, } \\
\text { ageing }\end{array}$ & 3 & $\begin{array}{l}\text { Design } \\
\text { controls } \\
\text { have an } \\
\text { even } \\
\text { chances }\end{array}$ & 6.3 & 105 & & 2 & 2 & 2 & 8 \\
\hline
\end{tabular}


International Journal of Engineering Applied Sciences and Technology, 2021

Vol. 5, Issue 10, ISSN No. 2455-2143, Pages 44-54

Published Online February 2021 in IJEAST (http://www.ijeast.com)

Table 5: FMECA of microcontroller unit

\begin{tabular}{|c|c|c|c|c|c|c|c|c|c|c|c|}
\hline \multirow[b]{2}{*}{$\begin{array}{l}\text { Name of } \\
\text { Unit/function }\end{array}$} & \multirow{2}{*}{$\begin{array}{l}\text { Failure } \\
\text { Mode }\end{array}$} & \multirow{2}{*}{$\begin{array}{l}\text { Effects(s } \\
\text { ) of } \\
\text { Failure }\end{array}$} & \multicolumn{6}{|c|}{ Risk rating } & \multirow[b]{2}{*}{$\begin{array}{l}\text { Actions } \\
\text { Taken }\end{array}$} & \multicolumn{2}{|l|}{ Revised risk } \\
\hline & & & $\mathbf{S}$ & $\begin{array}{l}\text { Cause(s) } \\
\text { of Failure }\end{array}$ & $\mathbf{O}$ & $\begin{array}{l}\text { Fault } \\
\text { Detectio } \\
\text { n }\end{array}$ & D & RPN & & $\begin{array}{lll}S & O & D\end{array}$ & $\begin{array}{l}\mathbf{R} \\
\mathbf{P} \\
\mathbf{N}\end{array}$ \\
\hline $\begin{array}{l}\text { Microcontroll } \\
\text { er / Interlink } \\
\text { the units and } \\
\text { house the } \\
\text { software }\end{array}$ & $\begin{array}{l}\text { Output } \\
\text { struck, } \\
\text { input } \\
\text { struck, } \\
\text { drift of } \\
\text { frequen } \\
\text { cy }\end{array}$ & $\begin{array}{l}\text { Leads to } \\
\text { entire } \\
\text { system } \\
\text { failure }\end{array}$ & 8 & $\begin{array}{l}\text { Manufact } \\
\text { urer } \\
\text { defect, } \\
\text { static } \\
\text { charges }\end{array}$ & 4 & $\begin{array}{l}\text { Extremel } \\
\mathrm{y} \\
\text { Unlikely }\end{array}$ & 10 & 320 & $\begin{array}{l}\text { Compo } \\
\text { nent } \\
\text { earthing }\end{array}$ & $\begin{array}{lll}8 & 1 & 10\end{array}$ & $\begin{array}{l}8 \\
0\end{array}$ \\
\hline
\end{tabular}

Table 6: FMECA of software

\begin{tabular}{|c|c|c|c|c|c|c|c|c|c|c|c|c|c|}
\hline \multirow{2}{*}{$\begin{array}{l}\text { Name of } \\
\text { Unit/function }\end{array}$} & \multirow{2}{*}{$\begin{array}{l}\text { Failure } \\
\text { Mode }\end{array}$} & \multirow{2}{*}{$\begin{array}{l}\text { Effects(s } \\
\text { ) of } \\
\text { Failure }\end{array}$} & \multirow[b]{2}{*}{$\mathbf{S}$} & \multicolumn{4}{|c|}{ Risk rating } & \multirow[b]{2}{*}{ RPN } & \multirow[b]{2}{*}{$\begin{array}{l}\text { Actions } \\
\text { Taken }\end{array}$} & \multicolumn{4}{|c|}{ Revised risk } \\
\hline & & & & $\begin{array}{l}\text { Cause(s) } \\
\text { of Failure }\end{array}$ & $\mathbf{O}$ & $\begin{array}{l}\text { Fault } \\
\text { Detectio } \\
\text { n }\end{array}$ & D & & & $\mathbf{S}$ & $\mathbf{O}$ & D & $\begin{array}{l}\mathbf{R P} \\
\mathbf{N}\end{array}$ \\
\hline \multirow{3}{*}{$\begin{array}{l}\text { Software ( } \\
\text { Mikro C) / } \\
\text { Responsible } \\
\text { control of the } \\
\text { entire system }\end{array}$} & $\begin{array}{l}\text { Data } \\
\text { related } \\
{[17]}\end{array}$ & $\begin{array}{l}\text { System } \\
\text { failure }\end{array}$ & 8 & $\begin{array}{l}\text { Software } \\
\text { designer } \\
\text { defect }\end{array}$ & 4 & $\begin{array}{l}\text { Very } \\
\text { Low } \\
\text { Likelihoo } \\
\text { d }\end{array}$ & 9 & 288 & $\begin{array}{l}\text { hardwar } \\
\text { e } \\
\text { redunda } \\
\text { ncy, }\end{array}$ & 5 & 2 & 9 & 90 \\
\hline & $\begin{array}{l}\text { Event } \\
\text { related } \\
{[17]}\end{array}$ & $\begin{array}{l}\text { System } \\
\text { failure }\end{array}$ & 8 & $\begin{array}{l}\text { Software } \\
\text { designer } \\
\text { defect }\end{array}$ & 4 & $\begin{array}{l}\text { Very } \\
\text { Low } \\
\text { Likelihoo } \\
\text { d }\end{array}$ & 9 & 288 & $\begin{array}{l}\text { effectiv } \\
\text { e design } \\
\text { and } \\
\text { code } \\
\text { reviews }\end{array}$ & 5 & 2 & 9 & 90 \\
\hline & Average & & 8 & & 4 & & 9 & 288 & & 5 & 2 & 9 & 90 \\
\hline
\end{tabular}

Table 7: FMECA of RFID system

\begin{tabular}{|c|c|c|c|c|c|c|c|c|c|c|c|c|c|}
\hline \multirow{2}{*}{$\begin{array}{l}\text { RFID } \\
\text { system }\end{array}$} & \multirow[t]{2}{*}{ Failure Mode } & \multirow{2}{*}{$\begin{array}{l}\text { Effects } \\
\text { (s) of } \\
\text { Failure }\end{array}$} & \multicolumn{6}{|c|}{ Risk rating } & \multirow[b]{2}{*}{$\begin{array}{l}\text { Action } \\
\text { s } \\
\text { Taken }\end{array}$} & \multicolumn{4}{|c|}{ Revised risk } \\
\hline & & & $\begin{array}{l}\text { S } \\
\text { E } \\
\text { V }\end{array}$ & $\begin{array}{l}\text { Cause(s) } \\
\text { of Failure }\end{array}$ & $\begin{array}{l}\text { OC } \\
\text { C }\end{array}$ & $\begin{array}{l}\text { Fault } \\
\text { Detecti } \\
\text { on }\end{array}$ & $\begin{array}{l}\text { DE } \\
T\end{array}$ & $\begin{array}{l}\mathbf{R P} \\
\mathbf{N}\end{array}$ & & $\mathbf{S}$ & $\mathbf{O}$ & D & $\begin{array}{l}\mathbf{R} \\
\mathbf{P} \\
\mathbf{N}\end{array}$ \\
\hline $\begin{array}{l}\text { Tag } \\
\text { failure. }\end{array}$ & $\begin{array}{l}\text { Output struck, } \\
\text { drift of } \\
\text { frequency }\end{array}$ & $\begin{array}{l}\text { System } \\
\text { failure }\end{array}$ & 8 & $\begin{array}{l}\text { Physical, } \\
\text { Virus } \\
\text { attack, }\end{array}$ & 5 & $\begin{array}{l}\text { High } \\
\text { Likelih } \\
\text { ood }\end{array}$ & 3 & 120 & $\begin{array}{l}\text { Kept } \\
\text { hidden }\end{array}$ & 8 & 1 & 3 & 24 \\
\hline $\begin{array}{l}\text { Failure } \\
\text { of reader }\end{array}$ & $\begin{array}{l}\text { input struck, } \\
\text { drift of } \\
\text { frequency }\end{array}$ & $\begin{array}{l}\text { System } \\
\text { failure }\end{array}$ & 8 & $\begin{array}{l}\text { Physical, } \\
\text { Virus } \\
\text { attack, } \\
\text { cloning, } \\
\text { eavesdrop } \\
\text { ping }\end{array}$ & 6 & $\begin{array}{l}\text { Modera } \\
\text { tely } \\
\text { High } \\
\text { Likelih } \\
\text { ood }\end{array}$ & 4 & 192 & & 8 & 2 & 4 & $\begin{array}{l}6 \\
4\end{array}$ \\
\hline & Average & & 8 & & 5.5 & & 3.5 & 156 & & 8 & 1.5 & 3.5 & 44 \\
\hline
\end{tabular}

Table 8: FMECA of GSM module unit

GSM Failure Mode Effects

Risk rating

Revised risk 


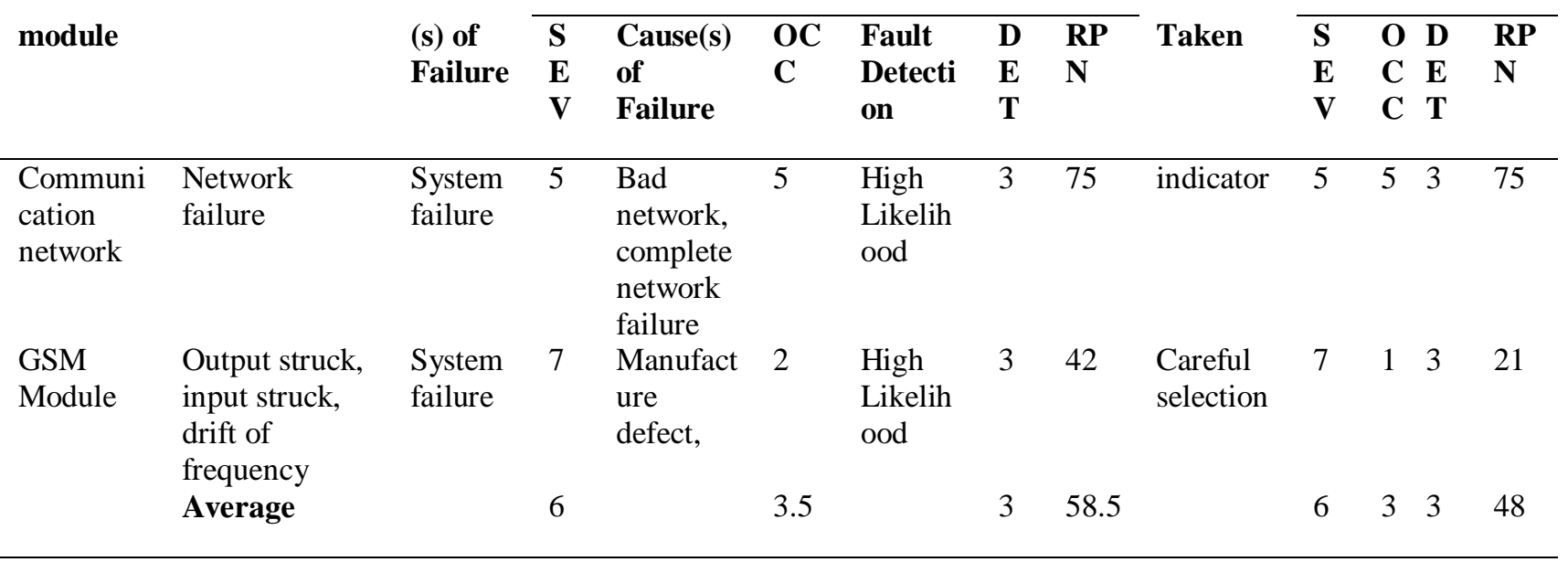

Table 9: FMECA of keypad unit

\begin{tabular}{|c|c|c|c|c|c|c|c|c|c|c|c|c|}
\hline \multirow{2}{*}{$\begin{array}{l}\text { Name of } \\
\text { Unit/function }\end{array}$} & \multirow{2}{*}{$\begin{array}{l}\text { Failure } \\
\text { Mode }\end{array}$} & \multirow{2}{*}{$\begin{array}{l}\text { Effects(s } \\
\text { ) of } \\
\text { Failure }\end{array}$} & \multicolumn{6}{|c|}{ Risk rating } & & \multicolumn{3}{|c|}{ Revised risk } \\
\hline & & & $\begin{array}{l}\text { SE } \\
\text { V }\end{array}$ & $\begin{array}{l}\text { Cause(s) } \\
\text { of Failure }\end{array}$ & $\begin{array}{l}\mathbf{O} \\
\mathbf{C} \\
\mathbf{C}\end{array}$ & $\begin{array}{l}\text { Fault } \\
\text { Detectio } \\
\text { n }\end{array}$ & $\begin{array}{l}\mathbf{D} \\
\mathbf{E} \\
\mathbf{T}\end{array}$ & $\begin{array}{l}\mathbf{R} \\
\mathbf{P} \\
\mathbf{N}\end{array}$ & $\begin{array}{l}\text { Actions } \\
\text { Taken }\end{array}$ & $\begin{array}{ll}\mathbf{S} & \mathbf{O} \\
\mathbf{E} & \mathbf{C} \\
\mathrm{V} & \mathbf{C}\end{array}$ & $\begin{array}{l}\mathbf{D} \\
\mathbf{E} \\
\mathbf{T}\end{array}$ & $\begin{array}{l}\mathbf{R P} \\
\mathbf{N}\end{array}$ \\
\hline $\begin{array}{l}\text { Key pad / } \\
\text { provide access } \\
\text { to key-in the } \\
\text { password. }\end{array}$ & $\begin{array}{l}\text { Open } \\
\text { circuit, } \\
\text { short } \\
\text { circuit }\end{array}$ & $\begin{array}{l}\text { System } \\
\text { failure }\end{array}$ & 8 & $\begin{array}{l}\text { Manufact } \\
\text { urer } \\
\text { defect, } \\
\text { ageing }\end{array}$ & 3 & $\begin{array}{l}\text { Moderate } \\
\text { ly High } \\
\text { Likeliho } \\
\text { od }\end{array}$ & 4 & 96 & $\begin{array}{l}\text { Careful } \\
\text { selection }\end{array}$ & $\begin{array}{ll}8 & 2\end{array}$ & 4 & 64 \\
\hline
\end{tabular}

Table 10: FMECA of lock unit

\begin{tabular}{|c|c|c|c|c|c|c|c|c|c|c|c|c|}
\hline \multirow[b]{2}{*}{$\begin{array}{l}\text { Name of } \\
\text { Unit/function }\end{array}$} & \multirow{2}{*}{$\begin{array}{l}\text { Failure } \\
\text { Mode }\end{array}$} & \multirow{2}{*}{$\begin{array}{l}\text { Effects } \\
\text { (s) of } \\
\text { Failure }\end{array}$} & \multicolumn{6}{|c|}{ Risk rating } & \multirow[b]{2}{*}{$\begin{array}{l}\text { Actions } \\
\text { Taken }\end{array}$} & \multicolumn{3}{|c|}{ Revised risk } \\
\hline & & & $\begin{array}{l}\mathbf{S} \\
\mathbf{E} \\
\mathbf{V}\end{array}$ & $\begin{array}{l}\text { Cause(s) } \\
\text { of Failure }\end{array}$ & $\mathbf{O}$ & $\begin{array}{l}\text { Fault } \\
\text { Detec } \\
\text { tion }\end{array}$ & & $\begin{array}{l}\mathbf{R P} \\
\mathbf{N}\end{array}$ & & $\begin{array}{ll}\mathbf{S} & \mathbf{O} \\
\mathbf{E} & \mathbf{C} \\
\mathbf{V} & \mathbf{C}\end{array}$ & $\begin{array}{l}\mathbf{D} \\
\mathbf{E} \\
\mathbf{T}\end{array}$ & $\begin{array}{l}\mathbf{R P} \\
\mathbf{N}\end{array}$ \\
\hline $\begin{array}{l}\text { The lock or } \\
\text { motor /opens } \\
\text { or lock the } \\
\text { system }\end{array}$ & $\begin{array}{l}\text { Windin } \\
\mathrm{g} \\
\text { Failure } \\
\text { In short } \\
\text { Mode }\end{array}$ & $\begin{array}{l}\text { System } \\
\text { failure }\end{array}$ & 8 & $\begin{array}{l}\text { Low } \\
\text { starting } \\
\text { torque, } \\
\text { Misalign } \\
\text { ment of } \\
\text { teeth, } \\
\text { worn out }\end{array}$ & 5 & $\begin{array}{l}\text { High } \\
\text { Likeli } \\
\text { hood }\end{array}$ & 3 & 120 & $\begin{array}{l}\text { Current limit } \\
\text { circuit introduced. } \\
\text { Redundant motor } \\
\text { and redundant } \\
\text { winding to be } \\
\text { introduced. }\end{array}$ & $\begin{array}{ll}8 & 3\end{array}$ & 3 & 72 \\
\hline
\end{tabular}

Table 11: FMECA of the lock system

\begin{tabular}{|c|c|c|c|c|c|c|c|c|}
\hline \multirow{2}{*}{ Name of system } & \multicolumn{4}{|c|}{ Risk rating } & \multicolumn{4}{|c|}{ Revised risk } \\
\hline & $\mathbf{S}$ & $\mathbf{O}$ & D & RPN & $\mathbf{S}$ & $\mathbf{O}$ & D & RPN \\
\hline lock & 7.69 & 4.14 & 5.54 & 182.11 & 6.43 & 2.07 & 4.93 & 58 \\
\hline $\begin{array}{l}\text { IV. RESULT AND } \\
\text { The result of FMECA of each } \\
\text { system shows an improven } \\
\text { reliability. For the power sup }\end{array}$ & $\begin{array}{l}\text { that } \\
\text { in } \\
\text { unit, }\end{array}$ & $\begin{array}{c}u_{1} \\
\text { sy } \\
\text { ery }\end{array}$ & & \multicolumn{5}{|c|}{$\begin{array}{l}\text { used as a backup which changes severance from } 7.75 \\
\text { to } 2 \text {, occurrence from } 4 \text { to } 2 \text {, detection from } 6.25 \text { to } 2 \text {, } \\
\text { and risk priority number (RPN) from } 236.25 \text { to. It } \\
\text { thus reduces the severance if failure occur, possible } \\
\text { occurrence and likelihood of failure detection by } \\
74 \%, 50 \% \text { and } 68 \% \text { respectively. Subsequently the } \\
\text { risk priority number (RPN) reduces by } 97 \% \text { as shown }\end{array}$} \\
\hline
\end{tabular}


in figure 2.
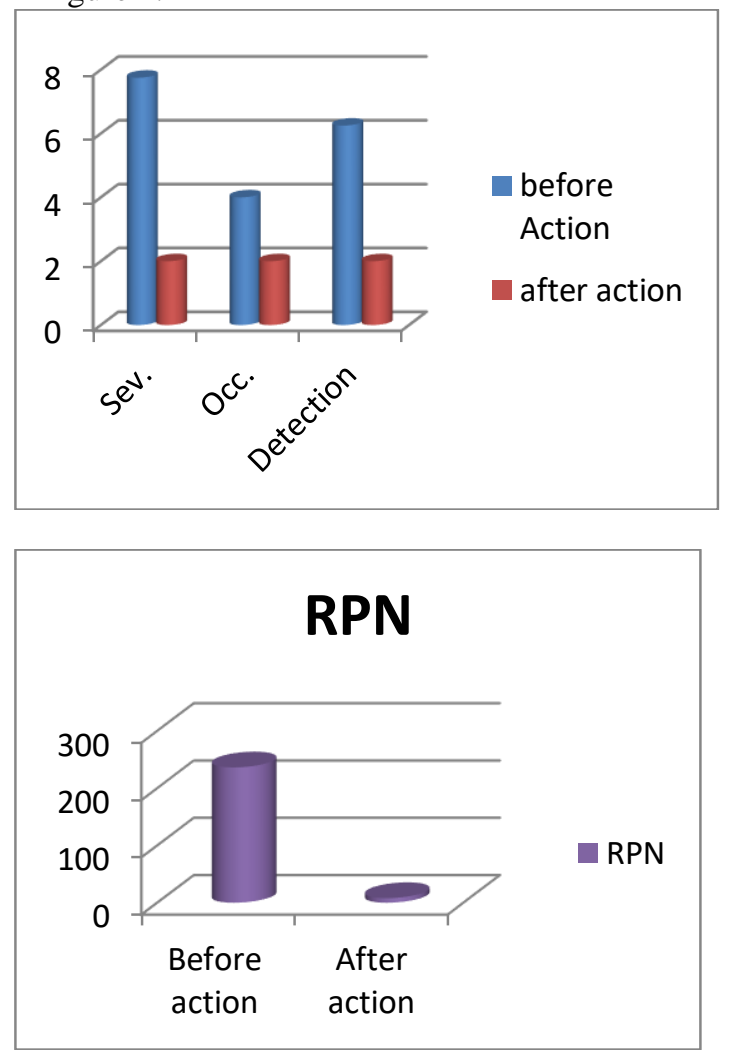

Figure 2: FMECA of power supply unit

In the microcontroller unit, Component earthing, careful selection of microcontroller was the action taken which changes only occurrence from 4 to1 and risk priority number (RPN) from 320 to 80 . It thus reduces only the possible occurrence of failure by $75 \%$ whiles the severance and detection if failure occur remains unchanged. The risk priority number (RPN) also reduces by $75 \%$ as shown in figure 3 .

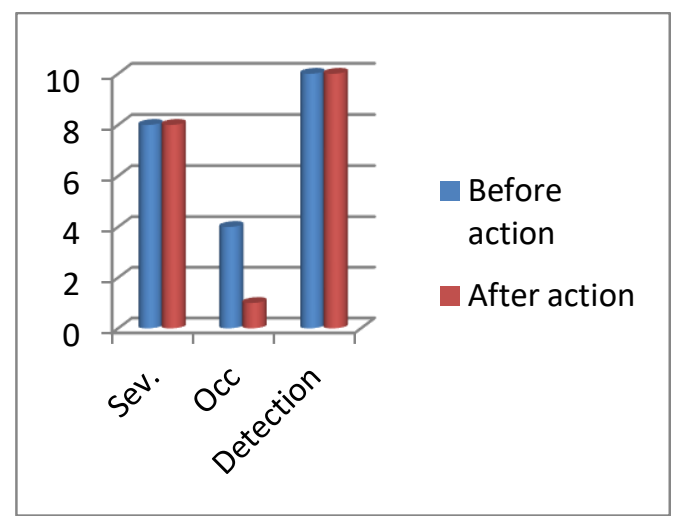

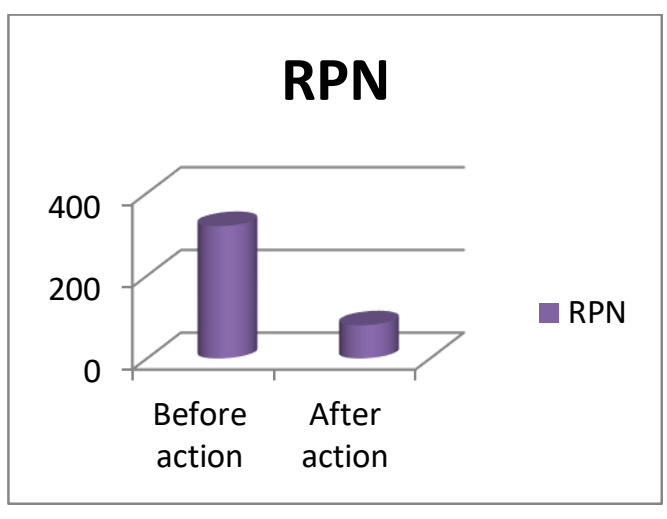

Figure 3: FMECA of microcontroller unit

To increase the reliability of the system, hardware redundancy, effective design and code reviews were the measures taken to increase the reliability of the software which changes the severance from 8 to 5 occurrence from 4 to 2 and risk priority number (RPN) from 288 to 90 . This reduces the severance if failure occurs in the software by $37.5 \%$, possible occurrence by $50 \%$ but the likelihood detection of such failure remains unchanged remains unchanged. Subsequently the risk priority number (RPN) reduces by $68.75 \%$ as shown in figure 4 .
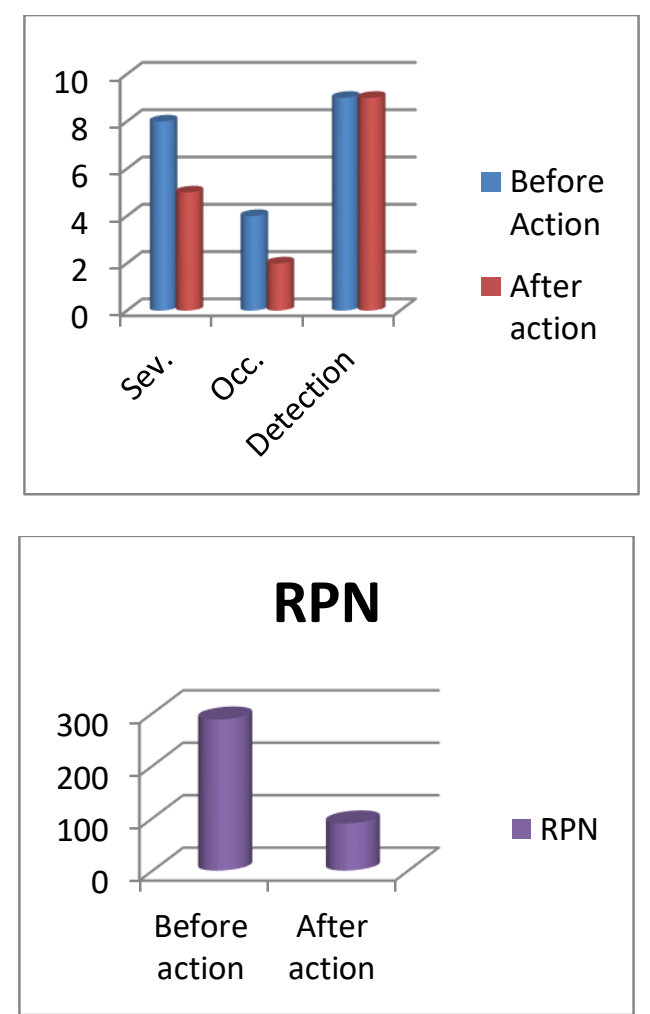
Figure 4: FMECA of the software

For the RFID system: Physical, Virus attack, cloning and eavesdropping were the possible causes of failure. The RFID system (Tag and Reader) kept hidden which changes only occurrence from 5.5 to 1.5 and risk priority number (RPN) from 156 to 44 . It thus reduces the possible occurrence of failure by $\sim 73 \%$ while the severance if it occurs and likelihood of detection remains unchanged. The risk priority number (RPN) reduces by $\sim 72 \%$ as shown in figure 5.
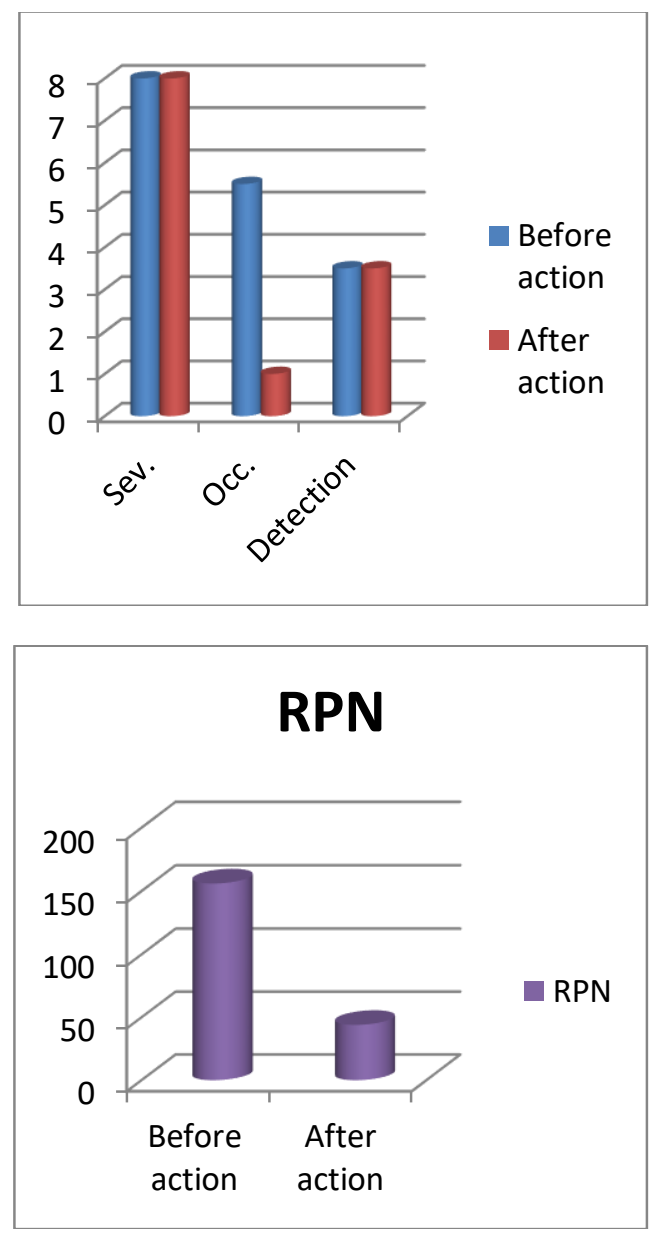

Figure 5: FMECA of RFID system

For the GSM module unit: Bad network, complete network failure and Manufacture defect, will have been the likely causes of failure. Careful selection was adopted to avert the latter and a light emitting diode (LED) to indicate when a command is given to the GSM module. These changes the occurrence from 3.5 to 3 detection from 3 to 1 and risk priority number (RPN) from 63 to 18 . Thus, reduce the possible occurrence of failure by $\sim 43 \%$ and the likelihood of detection by $67 \%$ while the severance remains unchanged. The risk priority number (RPN) also reduces by $\sim 76 \%$ as shown in figure 6 .
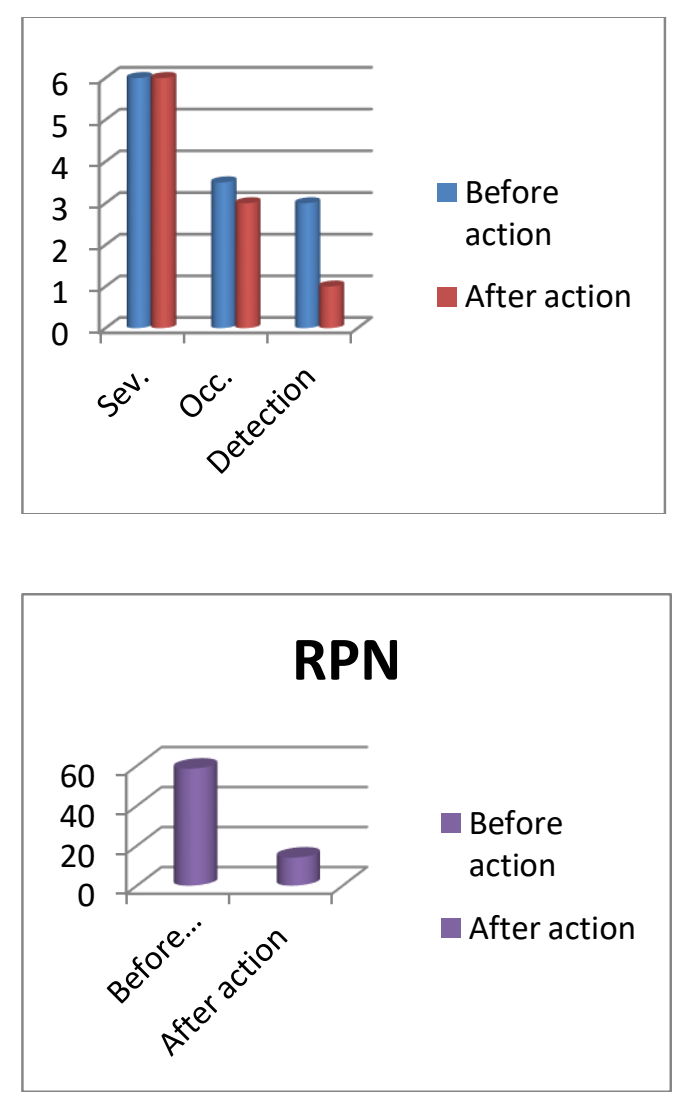

Figure 6: FMECA of GSM module unit

To reduce the failure rate of the system, careful selection of keypad was the measure taken in the keypad unit. This changes the occurrence from 3 to 2 and risk priority number (RPN) from 96 to 64. This reduces the possible occurrence of failure of the unit by $\sim 33 \%$ but the severance if failure occurred and likely detection of such failure remain unchanged. Subsequently the risk priority number (RPN) also reduces by $33 \%$ as shown in figure 7 . 

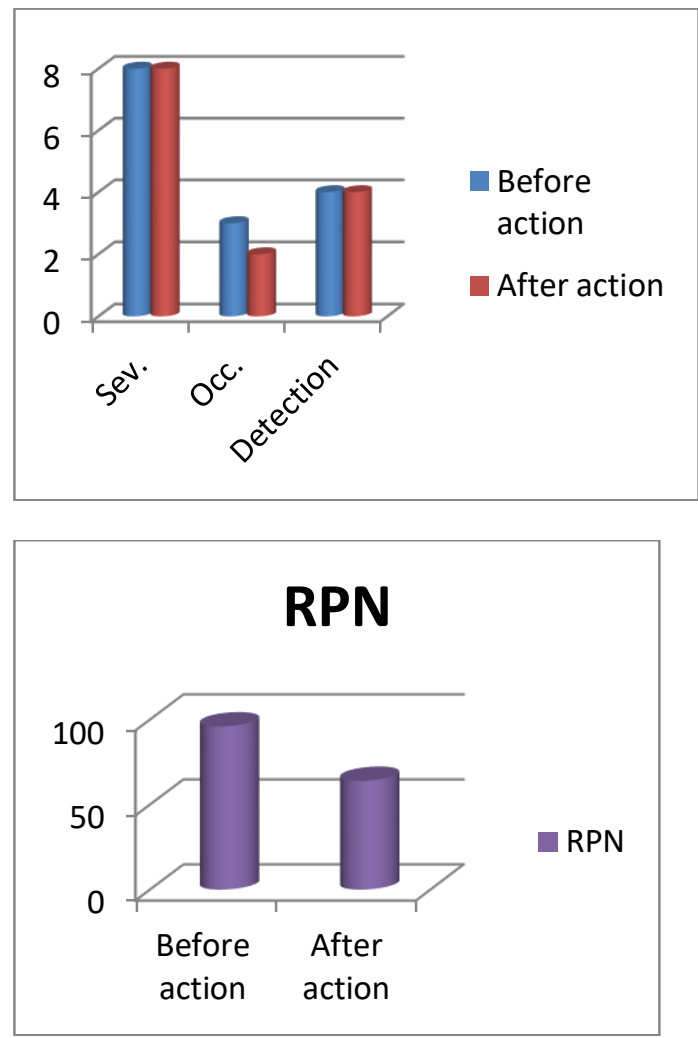

Figure 7: FMECA of keypad unit

High starting torques, Misalignment of teeth and worn out due to friction were predicted to be likely cause of failure. Lubrication is adopted to avert these which change the occurrence from 5 to 3 and risk priority number (RPN) from 120 to 72 . Thus, rate of occurrence of fault is expected to reduce by $40 \%$. The RPN also reduces by $40 \%$ as shown in figure 8 .

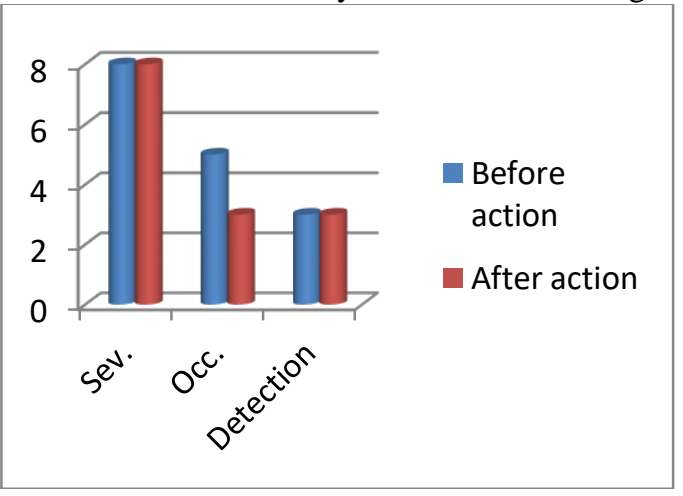

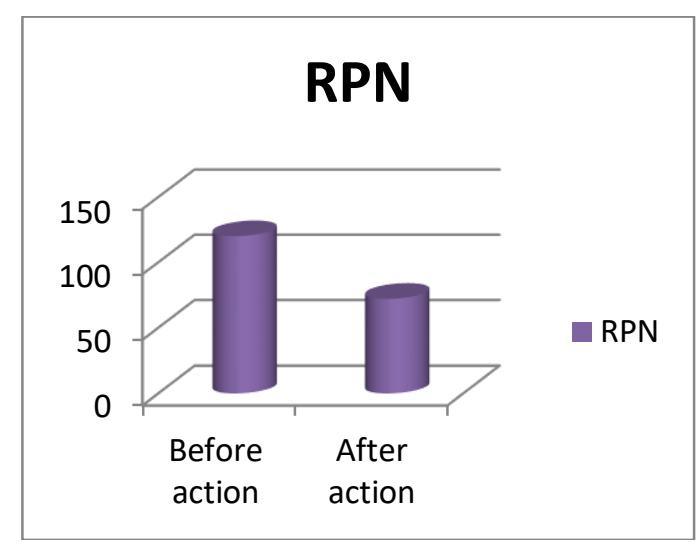

Figure 8: FMECA of lock system

With all the measures taken to increase the reliability of the system, the severance changes from 7.69 to 6.43, occurrence from 4.14 to 2.07 , detection from 5.54 to 4.93 and risk priority number (RPN) from 182.11 to 58 . Thus, the severance of the system due to failure reduces by $\sim 16.4$, the possible occurrence by $50 \%$ and the likely detection of such failure by $\sim 11 \%$. The RPN also reduces by $\sim 68 \%$ as shown in figure 9 .

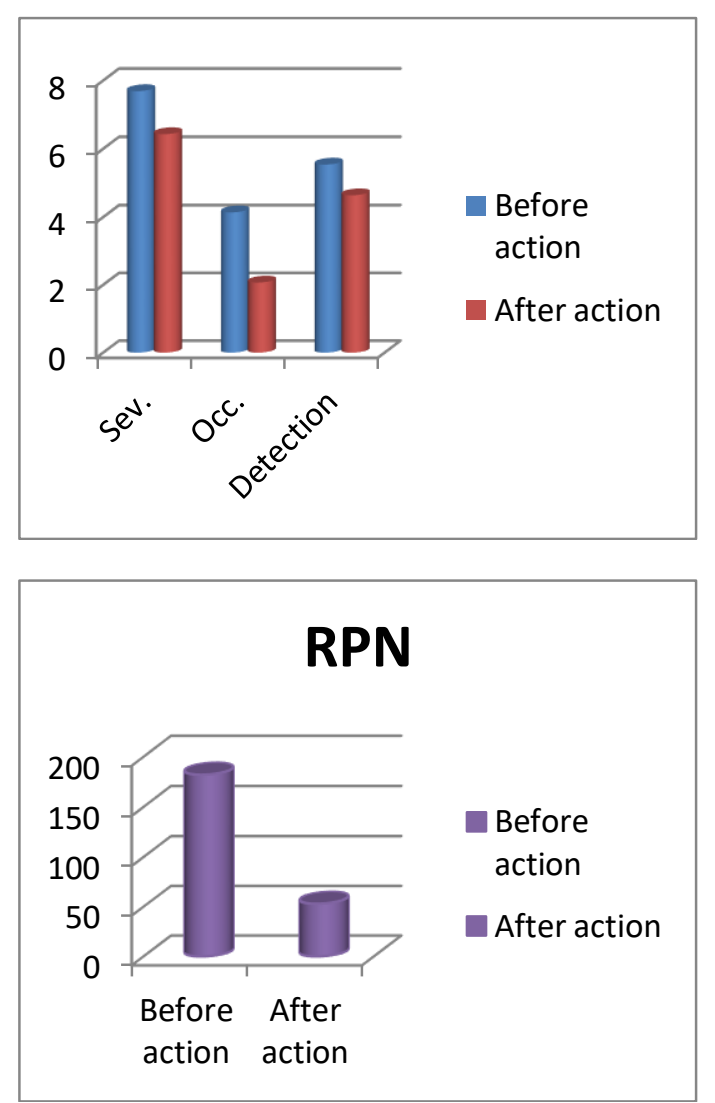




\section{International Journal of Engineering Applied Sciences and Technology, 2021 \\ Vol. 5, Issue 10, ISSN No. 2455-2143, Pages 44-54 \\ Published Online February 2021 in IJEAST (http://www.ijeast.com)}

Figure 9: FMECA of the system

\section{CONCLUSION}

In this paper background information on an RFID-GSM based Lock System, and FMECA was presented. Reliability analysis has been conducted by defining the unit/component parts, identifying the failure modes, Effects(s) of Failure, Cause(s) of Failure and detection of fault. An action has been taken to improve the system reliability and a number has been allotted depending on the degree for severance, occurrence and detection and subsequently the RPN was calculated for both before and after the action. As shown in the result, the system reliability improved. While it is quite unlikely that any system can be made $100 \%$ reliable, identifying the failure modes and taking an action to increase the system reliability are vital steps toward improving any system reliability.

\section{REFERENCES}

[1] Sellappan N. and Palanikumar K. (2013) "Modified Prioritization Methodology for Risk Priority Number in Failure Mode and Effects Analysis" International Journal of Applied Science and Technology Vol. 3 No. 4pp 27-36

[2] Tamer M. E., Ahmed M. E., Islam H. A. and Ahmed A.(2016), "Implementation of FMECA and Fishbone Techniques in Reliability Centered Maintenance Planning" International Journal of Innovative Research in Science, Engineering and Technology, Vol. 5, Issue 11 pp 1801-1811

[3]

http://media.futuresmag.com/futuresmag/arti cle/2012/07/05/istock_000019129829XSmal l- resize-380x300.jpg

[4] Illiashenko O. \& Babeshko E (2012): "choosing FMECA-based techniques and tools for safety analysis of critical systems" Information and security, An international journal, vol 28, No 2, pp 275-285

[5] Shwetha K, Narahari N. S. \& Chandra S. P. (2013), "Failure Mode Effect and Criticality Analysis Performance Test on DC Brush Motors Used in Spacecraft Applications" International Journal of Research in
Engineering \& Technology (IJRET) Vol. 1, Issue 2, pp 169-176

[6] Omar N. S. et al, (2017), "Analysis of Failure Modes Effect and Criticality Analysis (FMECA): A Stand-Alone Photovoltaic System", Science Journal of Energy Engineering. Vol. 5, No. 2, pp. 4047. doi:10.11648/j.sjee.20170502.11

[7] Fateme D., Babakalli A. Mahmood S. Christophe B. and Ashraf L. (2016) "Risk Evaluation of Railway Rolling Stock Failures Using FMECA Technique: A Case Study of Passenger Door System" Urban Rail Transit DOI10.1007/s40864-016-0043$\mathrm{z}$ www.springerlink.com

[8] Deeptesh S. and Amit S. (2015) "Study of Centrifugal Pump Using Failure Mode Effect and Critical Analysis Based on Fuzzy Cost Estimation: A Case Study" International Journal of Science and Research (IJSR) Volume 4 Issue 7, pp 19-22 www.ijsr.net

[9] Mohsen A., Khazaee P. Sabetghadam I., and Karimifard P. (2013) "Failure Modes and Effects Analysis (FMEA) for Power Transformers" paper presented at 28th Power System Conference - 2013 Tehran, Iran

[10] Denny R. S., Susan E. R., Stephanie A. F., Stephen M. R., and Paul E. G. (2009) "Failure Modes and Effects Analysis for Hydrogen Fuel Cell Vehicles - Subtask 1" Battelle Memorial Institute 505 King Ave Columbus, OH 43201

[11] Tejaskumar S. P. and Mihir T. P (2014) "A Case Study: A Process FMEA Tool to Enhance Quality and Efficiency of Manufacturing Industry" Bonfring International Journal of Industrial Engineering and Management Science, Vol. 4, No. 3 pp145-152

Ebeling, C. (2010), “ Intro to Reliability \& Maintainability Engineering," 2nd ed. Waveland Press, Inc.

[13] Akbari M, Khazaee P. Sabetghadam I. and Karimifard P. (2013) "Failure Modes and 
Effects Analysis (FMEA) for Power Transformers" $28^{\text {th }}$ international power system conference Tehran Iran

[14] SYDNEY WATER (2010), "Failure Mode Effects and Criticality Analysis (FMECA)", Document Owned by Manager, Strategic Asset Management BMIS Number: AMQ0006 Version 03 Issue Date: June Page 1-11

[15] Final Report of FMECA analysis for Battery Charger board prepared for AEi Systems, LLC 5933 W. Century Blvd. Suite 1100 Los Angeles CA 90045 (2006)

[16] ANNEX 6: EEE Components Failure Modes for FMECA

[17] Ann M. N. (2010) "Software Failure Modes Effects Analysis Overview" SoftRel, LLC pp 1-26 www.softrel.com amneufelder@softrel.com 